\title{
INTERPOLATED FINITE ELEMENT METHOD FOR SOME FOURTH-ORDER ELLIPTIC PROBLEMS
}

\author{
ANDREY B. ANDREEV ${ }^{1}$ AND MILENA R. RACHEVA ${ }^{2}$ \\ Institute for Information and Communication Technology \\ Bulgarian Academi of Sciences \\ Sofia, BULGARIA \\ Department of Mathematics and Informatics \\ Technical University of Gabrovo \\ Gabrovo, BULGARIA
}

\begin{abstract}
We introduce nonstandard interpolated finite elements providing better accuracy for a fourth-order elliptic boundary value problems, as well as to the biharmonic eigenvalue problems. The term "ultraconvergence" indicates that the convergence rate is at least two orders higher than the optimal global rate. This method is a variant of a postprocessing procedure when the known finite element solution is used. Moreover, a posteriori error estimates of global ultraconvergent type are derived.

The presented approach is applicable for the general rectangular finite element meshes. Some numerical results illustrate the efficiency of the proposed algorithm.
\end{abstract}

AMS Subject Classification: 65N30, 65N25, 65N12, 65N15, 65D10

Key Words: finite element method, ultraconvergence, superconvergence, fourthorder elliptic problems

Received: December 1, 2017; Accepted: April 20, 2018;

Published: May 21, $2018 \quad$ doi: $10.12732 /$ dsa.v27i2.13

Dynamic Publishers, Inc., Acad. Publishers, Ltd. https://acadsol.eu/dsa

\section{INTRODUCTION}

In recent years, superconvergence and ultraconvergence for finite element (FE) solutions have been an active area in numerical analysis and its applications. The main objective of this field of studies is to improve the existing approximation accuracy by applying certain postprocessing techniques which are relatively easy to implement. 
Zienkiewicz and J. Zhu [16, 17] developed an effective error estimation technique of finite element approximations. Their method falls into the category of recoverybased methods. This a posteriori error estimation technique approximates the error in energy or an energy norm on a particular partition. There are many ideas which are closely related to the recovery technique (see $[7,13]$ ). Mathematical proofs for the derivative patch recovery can be found in Li and Zhang [6]. For instance, there are proofs for some finite element methods under special assumptions of the partition and, in Q. Zhu and Zhao [15], for one-dimensional correction to the finite element solution. Relative references on a posteriori error estimation and adaptivity could be found in Ainsworth and Oden [1], and in Verfürth [11].

Our aim of this paper is to establish an ultraconvergence result for planar FE approximations of the elliptic fourth-order problem by using an interpolated FEM approach. Similar considerations are presented by Q. Lin et al. [7] for second order elliptic problems (see also [14]).

Supercloseness of a nonstandard nodal interpolant is exploited in this paper. This is done in order to develop postprocessing schemes to improve the approximation order of $\Delta u_{h}$ from $\mathcal{O}\left(h^{3}\right)$ to $\mathcal{O}\left(h^{5}\right)$, especially when polynomials of degree four in each variable are applied in FEM. An ultraconvergence result for fourth-order eigenvalue problems is obtained by means of a theorem proving the supercloseness between the Ritz projection and the corresponding approximate eigenfunction.

By applying the method ultraconvergent results are obtained without using higher elements. This procedure is based on a higher interpolation of the FE solution on the original finite elements.

Here follows the general layout of the paper: In Section 2, we present a review of a model for a fourth-order problem and its finite element procedure. In Section 3, we construct an auxiliary interpolation operator $i_{h}$, which verifies certain "verticesedges" conditions. The main body of the paper contains the proofs of superclosed properties, generated by the operator $i_{h}$. This is presented in Section 4. Section 5 is devoted to the construction of high interpolation $I_{2 h}$ and the proof of a global ultraconvergence error estimate. In Section 6, we show that the method yields an ultraconvergent scheme for a fourth-order eigenvalue problem. The paper closes by some numeric examples illustrating the theory and concluding remarks.

\section{SETTING OF THE PROBLEM AND FEM}

Let us consider the fourth-order elliptic model problem:

$$
\Delta^{2} u(x, y)=f(x, y), \quad(x, y) \in \Omega,
$$


with boundary conditions

$$
u(x, y)=\frac{\partial u}{\partial \nu}(x, y)=0, \quad(x, y) \in \Gamma
$$

where $\Omega \subset \mathbf{R}^{\mathbf{2}}$ is a simply connected polyrectangular domain with boundary $\Gamma$ and $\nu$ is the outher normal vector.

Let $H^{m}(\Omega)$ be the real Sobolev space for a nonnegative integer $m$ and their associated norms $\|\cdot\|_{m, \Omega}$ and seminorms $|\cdot|_{m, \Omega}$. The weak solution of the problem (1)-(2) is a function $u(x, y)$, belonging to $V \equiv H_{0}^{2}(\Omega)$,

$$
H_{0}^{2}(\Omega)=\left\{v \in H^{2}(\Omega):\left.v\right|_{\Gamma}=\left.\frac{\partial v}{\partial \nu}\right|_{\Gamma}=0\right\}
$$

and satisfying

$$
a(u, v)=(f, v) \quad \forall v \in V
$$

where

$$
a(u, v)=\int_{\Omega} \Delta u \Delta v d x d y
$$

and the notation $(\cdot, \cdot)$ is adopted for the $L_{2}(\Omega)$-inner product.

Obviously the $a$-form is symmetric and coercive on $V$, i.e.

$$
a(u, v)=a(v, u), \quad \rho\|v\|_{2, \Omega}^{2} \leq a(v, v), \quad \forall u, v \in V,
$$

where $\rho$ is a positive constant. Moreover, it is continuous:

$$
|a(u, v)| \leq C\|u\|_{2, \Omega}\|v\|_{2, \Omega}, \quad \forall u, v \in V .
$$

Henceforth, $C$ represents generic and positive constant.

Consider a family of regular partitions $\tau_{h}$ of $\bar{\Omega}$ consisting of rectangular finite elements $T$, which fulfill standard assumptions [5] and suppose that this family satisfies the inverse assumptions ([5], p. 140). Here $h$ is a FE parameter. We denote by $Q_{k}$ the set of polynomials of degree $k$ at most in each variable.

Let $V_{h} \subset V$ be finite element spaces associated with the partition $\tau_{h}$. Assume that the polynomial space in the construction of $V_{h}$ contains $Q_{n}, n \geq 3$. Our aim for constructing an ultraconvergent interpolant requires $n \geq 4$. In order to avoid technical difficulties we confine the considerations to the case $n=4$.

Then

$$
V_{h}=\left\{v \in C^{1}(\bar{\Omega}) \cap V: v_{\mid T} \in Q_{4}(T), \forall T \in \tau_{h}\right\} .
$$

Let $P_{h}: V \rightarrow V_{h}$ be the elliptic projection operator defined by

$$
a\left(u-P_{h} u, v\right)=0 \quad \forall u \in V, \forall v \in V_{h}
$$


It is well-known (see [11]) that if the solution $u(x, y)$ of $(3)$ belongs to $H^{5}(\Omega) \cap V$, then

$$
\left\|u-P_{h} u\right\|_{2, \Omega} \leq C h^{3}\|u\|_{5, \Omega} .
$$

We shall construct a nonstandard interpolation operator which improve the optimal estimate (4). Two principal steps are performed for this purpose. The first one is to prove a local supercloseness in energy norm by means of special interpolation operator $i_{h}$ and the second one is to prove a global ultraconvergence to $I_{2 h} P_{h} u$, where the operator $I_{2 h}$ verifies certain ultraconvergence properties.

\section{CONSTRUCTION OF THE OPERATOR $\mathbf{I}_{\mathbf{H}}$}

Consider the partition $\tau_{h}=\bigcup T$, where for any finite element $T$ its center is denoted by $\left(x_{T}, y_{T}\right)$ and its element dimensions in $x$ and $y$-direction are denoted by $h_{1, T}, h_{2, T}$, respectively. The following denotations are also adopted:

$$
h_{T}=\max \left(h_{1, T} ; h_{2, T}\right), \quad h=\max _{T \in \tau_{h}} h_{T} .
$$

Also, the vertices and edges of any element $T$ are noted by $s_{j}$ and $l_{j}, j=1, \ldots, 4$, respectively.

Taking in consideration the case $n=4$ we introduce the interpolation operator $i_{h}: C^{1}(T) \rightarrow V_{h}(T)$ using the so-called "vertices - edges" conditions: $\forall v \in C^{1}(T)$ and $j=1, \ldots, 4$

$$
\begin{array}{cc}
i_{h} v\left(s_{j}\right)=v\left(s_{j}\right), & \frac{\partial i_{h} v}{\partial x}\left(s_{j}\right)=\frac{\partial v}{\partial x}\left(s_{j}\right) \\
\frac{\partial i_{h} v}{\partial y}\left(s_{j}\right)=\frac{\partial v}{\partial y}\left(s_{j}\right), & \frac{\partial^{2} i_{h} v}{\partial x \partial y}\left(s_{j}\right)=\frac{\partial^{2} v}{\partial x \partial y}\left(s_{j}\right) \\
\int_{l_{j}} i_{h} v(x, y) d l_{j}=\int_{l_{j}} v(x, y) d l_{j}, & \int_{l_{j}} \frac{\partial i_{h} v}{\partial \nu}(x, y) d l_{j}=\int_{l_{j}} \frac{\partial v}{\partial \nu}(x, y) d l_{j}, \\
\int_{T} i_{h} v(x, y) d x d y=\int_{T} v(x, y) d x d y . &
\end{array}
$$

It is evident that $i_{h} v \equiv v$ for any $v \in V_{h}$. Let us remark that

$$
\left\|i_{h} v-v\right\|_{2, \Omega} \leq C h^{3}\|v\|_{5, \Omega}
$$

In one-dimensional case (see [3]) the basis determining the operator $i_{h}$ is $\left\{\varphi_{i}(t)\right\}_{i=1}^{5}$, $t \in[-1,1]$, where:

$$
\left(\begin{array}{l}
\varphi_{1}(t) \\
\varphi_{2}(t) \\
\varphi_{3}(t) \\
\varphi_{4}(t) \\
\varphi_{5}(t)
\end{array}\right)=\frac{1}{16}\left(\begin{array}{rrrrr}
-15 & 4 & 30 & -12 & -7 \\
-5 & 4 & 6 & -4 & -1 \\
15 & 0 & -30 & 0 & 15 \\
-15 & -4 & 30 & 12 & -7 \\
5 & 4 & -6 & -4 & 1
\end{array}\right)\left(\begin{array}{l}
t^{4} \\
t^{3} \\
t^{2} \\
t \\
1
\end{array}\right) .
$$


Then, for the two-dimensional case the basis is obtained by the products $\varphi_{i}(x)$. $\varphi_{s}(y), i, s=1, \ldots, 5$, i.e. is $\left\{\psi_{j}(x, y)\right\}_{j=1}^{25}$. Moreover,

$$
\left.i_{h} v(x, y)\right|_{T}=\sum_{j=1}^{25} \hat{v}_{j} \cdot \psi_{j}\left(\frac{x-x_{T}}{h_{1, T}}, \frac{y-y_{T}}{h_{2, T}}\right),
$$

where

$$
\begin{array}{cc}
\hat{v}_{4 i-3}=v\left(s_{i}\right), & \hat{v}_{4 i-2}=h_{1, T} \frac{\partial v}{\partial x}\left(s_{i}\right), \\
\hat{v}_{4 i-1}=h_{2, T} \frac{\partial v}{\partial y}\left(s_{i}\right), & \hat{v}_{4 i}=h_{1, T} h_{2, T} \frac{\partial^{2} v}{\partial x \partial y}\left(s_{i}\right), \quad i=1, \ldots, 4 ; \\
\hat{v}_{2 i+15}=\frac{1}{h_{1, T}} \int_{l_{i}} v(x, y) d x, & \hat{v}_{2 i+16}=\frac{h_{2, T}}{h_{1, T}} \int_{l_{i}} \frac{\partial v}{\partial y}(x, y) d x, \quad i=1 ; 3 ; \\
\hat{v}_{2 i+15}=\frac{1}{h_{2, T}} \int_{l_{i}} v(x, y) d y, & \hat{v}_{2 i+16}=\frac{h_{1, T}}{h_{2, T}} \int_{l_{i}} \frac{\partial v}{\partial x}(x, y) d y, \quad i=2 ; 4 ; \\
\hat{v}_{25}=\frac{1}{h_{1, T} h_{2, T}} \int_{T} v(x, y) d x d y .
\end{array}
$$

Remark 1. From (5) it is easy to see that $i_{h}$ satisfies the $C^{1}-$ condition. That is continuity of the function $i_{h} v$ and continuity of its first derivatives across the interelement boundaries.

\section{SUPERCLOSE PROPERTIES OF $\mathbf{I}_{\mathbf{H}}$}

This section presents important error estimates which are related to the interpolated finite element method. The difficulties for fourth-order elliptic problems arise from the availability of the mixed derivatives in the variational bilinear $a$-form. However,

$$
a(u, v)=\int_{\Omega}\left[\frac{\partial^{2} u}{\partial x^{2}} \frac{\partial^{2} v}{\partial x^{2}}+2 \frac{\partial^{2} u}{\partial x \partial y} \frac{\partial^{2} v}{\partial x \partial y}+\frac{\partial^{2} u}{\partial y^{2}} \frac{\partial^{2} v}{\partial y^{2}}\right] d x d y .
$$

This presentation is not unique in terms of subject-matter (see for example [10]).

Let us introduce the following error functions for any $T \in \tau_{h}$ :

$$
E(x)=\frac{1}{2}\left[\left(x-x_{T}\right)^{2}-h_{1, T}^{2}\right], \quad F(y)=\frac{1}{2}\left[\left(y-y_{T}\right)^{2}-h_{2, T}^{2}\right] .
$$

We use the identities:

$$
\begin{aligned}
x-x_{T} & =\frac{1}{90}\left[E^{3}(x)\right]^{(5)}, \\
\left(x-x_{T}\right)^{2} & =\frac{1}{1260}\left[E^{4}(x)\right]^{(6)}+\frac{h_{1, T}^{2}}{7} .
\end{aligned}
$$


Further, we use another pair of identities:

$$
\begin{aligned}
& \left(x-x_{T}\right)^{3}=\frac{1}{420}\left[E^{4}(x)\right]^{(5)}+\frac{h_{1, T}^{2}}{210}\left[E^{3}(x)\right]^{(5)}, \\
& \left(x-x_{T}\right)^{4}=\frac{1}{4725}\left[E^{5}(x)\right]^{(6)}+\frac{h_{1, T}^{2}}{1890}\left[E^{4}(x)\right]^{(6)}+\frac{h_{1, T}^{4}}{21} .
\end{aligned}
$$

The following Lemma gives some integral presentations by means of error function $E(x)$ :

Lemma 1. Let $v(x, y)$ be a sufficiently smooth function defined on $\bar{T}, T \in \tau_{h}$. Then (i) If $\int_{l_{i}} v(x, y) d y=\int_{l_{i}} \frac{\partial v}{\partial x}(x, y) d y=0, i=2 ; 4$, then

$$
\begin{aligned}
\int_{T}\left(x-x_{T}\right) v(x, y) d x d y= & -\frac{1}{90} \int_{T} E^{3}(x) \frac{\partial^{5} v}{\partial x^{5}}(x, y) d x d y \\
\int_{T}\left(x-x_{T}\right)^{3} v(x, y) d x d y= & -\frac{1}{420} \int_{T} E^{4}(x) \frac{\partial^{5} v}{\partial x^{5}}(x, y) d x d y \\
& -\frac{h_{1, T}^{2}}{210} \int_{T} E^{3}(x) \frac{\partial^{5} v}{\partial x^{5}}(x, y) d x d y
\end{aligned}
$$

(ii) If $\int_{l_{i}} v(x, y) d y=\int_{l_{i}} \frac{\partial v}{\partial x}(x, y) d y=\int_{T} v(x, y) d x d y=0, i=2 ; 4$, then

$$
\begin{aligned}
\int_{T}\left(x-x_{T}\right)^{2} v(x, y) d x d y= & \frac{1}{1260} \int_{T} E^{4}(x) \frac{\partial^{6} v}{\partial x^{6}}(x, y) d x d y \\
\int_{T}\left(x-x_{T}\right)^{4} v(x, y) d x d y= & \frac{1}{4725} \int_{T} E^{5}(x) \frac{\partial^{6} v}{\partial x^{6}}(x, y) d x d y \\
& +\frac{h_{1, T}^{2}}{1890} \int_{T} E^{4}(x) \frac{\partial^{6} v}{\partial x^{6}}(x, y) d x d y
\end{aligned}
$$

Proof. The four equalities (8) and (9) are proved by using identities (6) and (7) for the terms $\left(x-x_{T}\right)^{i}, i=1, \ldots, 4$. The Green's formula and the above conditions are applied.

Next, other identities related to the two error functions $E(x)$ and $F(y)$ are needed:

$$
\begin{aligned}
& \left(x-x_{T}\right)^{3}=\frac{1}{15}\left[E^{3}(x)\right]^{(3)}+\frac{3 h_{1, T}^{2}}{5}\left(x-x_{T}\right), \\
& \left(x-x_{T}\right)^{4}=\frac{1}{105}\left[E^{4}(x)\right]^{(4)}+\frac{6 h_{1, T}^{2}}{7}\left(x-x_{T}\right)^{2}-\frac{3 h_{1, T}^{4}}{35},
\end{aligned}
$$

and correspondingly

$$
\begin{aligned}
& \left(y-y_{T}\right)^{3}=\frac{1}{15}\left[F^{3}(y)\right]^{(3)}+\frac{3 h_{2, T}^{2}}{5}\left(y-y_{T}\right), \\
& \left(y-y_{T}\right)^{4}=\frac{1}{105}\left[F^{4}(y)\right]^{(4)}+\frac{6 h_{2, T}^{2}}{7}\left(y-y_{T}\right)^{2}-\frac{3 h_{2, T}^{4}}{35} .
\end{aligned}
$$


Lemma 2. Suppose that for sufficiently smooth function $v(x, y)$

$$
\begin{aligned}
& \frac{\partial^{i+j} v}{\partial x^{i} \partial y^{j}}\left(s_{k}\right)=0, \quad i, j=0,1 \\
& \int_{l_{k}} v d l=\int_{l_{k}} \frac{\partial v}{\partial \nu} d l=\int_{T} v d x d y=0, \quad k=1, \ldots, 4
\end{aligned}
$$

then the following six relations for the function $\left(x-x_{T}\right)^{i}\left(y-y_{T}\right)^{j} \frac{\partial^{4} v}{\partial x^{2} \partial y^{2}}$ when $i, j \in$ $\{0, \ldots, 4\}$ are valid:

$\left(\mathbf{R}_{1}\right)$ For $i, j=0,1,2$;

$$
\int_{T}\left(x-x_{T}\right)^{i}\left(y-y_{T}\right)^{j} \frac{\partial^{4} v}{\partial x^{2} \partial y^{2}} d x d y=0
$$

$\left(\mathbf{R}_{2}\right)$ For $j=0,1,2$ and, by analogy for $i=0,1,2$

$$
\begin{aligned}
& \int_{T}\left(x-x_{T}\right)^{3}\left(y-y_{T}\right)^{j} \frac{\partial^{4} v}{\partial x^{2} \partial y^{2}} d x d y=-\frac{1}{15} \int_{T} E^{3}(x)\left(y-y_{T}\right)^{j} \frac{\partial^{7} v}{\partial x^{5} \partial y^{2}} d x d y \\
& \int_{T}\left(x-x_{T}\right)^{i}\left(y-y_{T}\right)^{3} \frac{\partial^{4} v}{\partial x^{2} \partial y^{2}} d x d y=-\frac{1}{15} \int_{T} F^{3}(y)\left(x-x_{T}\right)^{i} \frac{\partial^{7} v}{\partial x^{2} \partial y^{5}} d x d y
\end{aligned}
$$

$\left(\mathbf{R}_{3}\right)$ For $j=0,1,2$ and $i=0,1,2$, respectively

$$
\begin{aligned}
& \int_{T}\left(x-x_{T}\right)^{4}\left(y-y_{T}\right)^{j} \frac{\partial^{4} v}{\partial x^{2} \partial y^{2}} d x d y=\frac{1}{105} \int_{T} E^{4}(x)\left(y-y_{T}\right)^{j} \frac{\partial^{8} v}{\partial x^{6} \partial y^{2}} d x d y \\
& \int_{T}\left(x-x_{T}\right)^{i}\left(y-y_{T}\right)^{4} \frac{\partial^{4} v}{\partial x^{2} \partial y^{2}} d x d y=\frac{1}{105} \int_{T} F^{4}(y)\left(x-x_{T}\right)^{i} \frac{\partial^{8} v}{\partial x^{2} \partial y^{6}} d x d y
\end{aligned}
$$

$\left(\mathbf{R}_{4}\right)$ For $i, j=3$

$$
\begin{aligned}
\int_{T}\left(x-x_{T}\right)^{3}\left(y-y_{T}\right)^{3} \frac{\partial^{4} v}{\partial x^{2} \partial y^{2}} d x d y= & \frac{1}{15} \int_{T} E^{3}(x)\left(y-y_{T}\right)^{3} \frac{\partial^{7} v}{\partial x^{5} \partial y^{2}} d x d y \\
& -\frac{h_{1, T}^{2}}{25} \int_{T} F^{3}(y)\left(x-x_{T}\right) \frac{\partial^{7} v}{\partial x^{2} \partial y^{5}} d x d y
\end{aligned}
$$

$\left(\mathbf{R}_{\mathbf{5}}\right)$ For $(i, j)=(3,4)$ and $(i, j)=(4,3)$, respectively

$$
\begin{gathered}
\int_{T}\left(x-x_{T}\right)^{4}\left(y-y_{T}\right)^{3} \frac{\partial^{4} v}{\partial x^{2} \partial y^{2}} d x d y=\frac{1}{105} \int_{T} E^{4}(x)\left(y-y_{T}\right)^{3} \frac{\partial^{7} v}{\partial x^{5} \partial y^{2}} d x d y \\
-\frac{2 h_{1, T}^{2}}{35} \int_{T}\left(x-x_{T}\right)^{2} F^{3}(y) \frac{\partial^{7} v}{\partial x^{2} \partial y^{5}} d x d y+\frac{h_{1, T}^{4}}{175} \int_{T} F^{3}(y) \frac{\partial^{7} v}{\partial x^{2} \partial y^{5}} d x d y \\
\int_{T}\left(x-x_{T}\right)^{3}\left(y-y_{T}\right)^{4} \frac{\partial^{4} v}{\partial x^{2} \partial y^{2}} d x d y=\frac{1}{105} \int_{T} F^{4}(y)\left(x-x_{T}\right)^{3} \frac{\partial^{7} v}{\partial x^{2} \partial y^{5}} d x d y \\
-\frac{2 h_{2, T}^{2}}{35} \int_{T}\left(y-y_{T}\right)^{2} E^{3}(x) \frac{\partial^{7} v}{\partial x^{5} \partial y^{2}} d x d y+\frac{h_{2, T}^{4}}{175} \int_{T} E^{3}(x) \frac{\partial^{7} v}{\partial x^{5} \partial y^{2}} d x d y
\end{gathered}
$$


$\left(\mathbf{R}_{6}\right)$ For $i, j=4$

$$
\begin{aligned}
\int_{T}(x- & \left.x_{T}\right)^{4}\left(y-y_{T}\right)^{4} \frac{\partial^{4} v}{\partial x^{2} \partial y^{2}} d x d y=\frac{1}{105} \int_{T} E^{4}(x)\left(y-y_{T}\right)^{4} \frac{\partial^{7} v}{\partial x^{5} \partial y^{2}} d x d y \\
& +\frac{2 h_{1, T}^{2}}{245} \int_{T}\left(x-x_{T}\right)^{2} F^{4}(y) \frac{\partial^{7} v}{\partial x^{2} \partial y^{5}} d x d y-\frac{h_{1, T}^{4}}{1225} \int_{T} F^{4}(y) \frac{\partial^{8} v}{\partial x^{2} \partial y^{6}} d x d y
\end{aligned}
$$

Proof. Direct calculations proves the first relation. The next one follows by presentations of $\left(x-x_{T}\right)^{3}$ and $\left(y-y_{T}\right)^{3}$ from (10), (11). Then integrating by parts and using $\left(R_{1}\right)$ we obtain $\left(R_{2}\right)$.

By way of analogy with the previous case the relation $\left(R_{3}\right)$ is connected with the presentation of $\left(x-x_{T}\right)^{4}$ and $\left(y-y_{T}\right)^{4}$ from (10), (11).

In order to prove the cases $\left(R_{4}\right)$ and $\left(R_{5}\right)$ we transform $\left(x-x_{T}\right)^{3}$ and $\left(x-x_{T}\right)^{4}$ by means of (10). Then, after integrating by parts we use the relation $\left(R_{2}\right)$.

The last case $\left(R_{6}\right)$ follows from the presentation of $\left(x-x_{T}\right)^{4}$ in (10) and from the corresponding equality of $\left(R_{3}\right)$.

Finally, the last two lemmas make it possible to formulate a superclose property concerning the interpolation operator $i_{h}$. This important result will be proved in the following theorem:

Theorem 1. Let the solution $u(x, y)$ of problem (3) belongs to the space $H^{7}(\Omega)$. Then, for any $v \in V_{h}(\Omega)$

$$
a\left(i_{h} u-u, v\right) \leq C h^{5}\|u\|_{7, \Omega}\|v\|_{2, \Omega} .
$$

Proof. Let us denote $U=i_{h} u-u$. The three ingredients of the $a$-form $a(U, v)$ have to be estimated. It should be noted that both terms

$$
\int_{\Omega} \frac{\partial^{2} U}{\partial x^{2}} \frac{\partial^{2} v}{\partial x^{2}} d x d y \text { and } \int_{\Omega} \frac{\partial^{2} U}{\partial y^{2}} \frac{\partial^{2} v}{\partial y^{2}} d x d y
$$

can be treated in a similar way.

Step 1. Consider $\int_{\Omega} \frac{\partial^{2} U}{\partial y^{2}} \frac{\partial^{2} v}{\partial y^{2}} d x d y, \forall v \in V_{h}$. So, for $(x, y) \in T$, the second factor is expanded as follows:

$$
\frac{\partial^{2} v}{\partial y^{2}}(x, y)=\sum_{s=0}^{4} \frac{\left(x-x_{T}\right)^{s}}{s !} \frac{\partial^{s+2} v}{\partial x^{s} \partial y^{2}}\left(x_{T}, y\right)
$$

taking into account that $\left.v(x, y)\right|_{T} \in Q_{4}(T)$, consequently

$$
\int_{\Omega} \frac{\partial^{2} U}{\partial y^{2}} \frac{\partial^{2} v}{\partial y^{2}} d x d y=\sum_{T \in \tau_{h}} \sum_{s=0}^{4} \int_{T} \frac{\left(x-x_{T}\right)^{s}}{s !} \frac{\partial^{2} U}{\partial y^{2}} \frac{\partial^{s+2} v}{\partial x^{s} \partial y^{2}}\left(x_{T}, y\right) d x d y
$$


The first term $(s=0)$ in the right-hand side of (13) is cancelled. This fact follows by definition of $U(x, y)$ and using Green's formula, i.e.

$$
\int_{T} \frac{\partial^{2} U}{\partial y^{2}} \frac{\partial^{2} v}{\partial y^{2}}\left(x_{T}, y\right) d x d y=0
$$

In order to transform the other components of (13), it has to be verified that the functions

$$
\frac{\partial^{2} U}{\partial y^{2}} \frac{\partial^{s+2} v}{\partial x^{s} \partial y^{2}}\left(x_{T}, y\right), \quad s=1, \ldots, 4,
$$

fulfill the conditions of Lemma 1 . Hence, the relations (8) and (9) will be satisfied for $s=1 ; 3$ and $s=2 ; 4$ respectively.

Let $i=2 ; 4$ and $s=1, \ldots, 4$. Integrating by parts reveals that:

$$
\begin{aligned}
\int_{l_{i}} \frac{\partial^{2} U}{\partial y^{2}} \frac{\partial^{s+2} v}{\partial x^{s} \partial y^{2}}\left(x_{T}, y\right) d y= & \left.\frac{\partial U}{\partial y}\left(x_{T} \pm h_{1, T}, y\right) \frac{\partial^{s+2} v}{\partial x^{s} \partial y^{2}}\left(x_{T}, y\right)\right|_{y_{T}-h_{2, T}} ^{y_{T}+h_{2, T}} \\
-\int_{l_{i}} \frac{\partial U}{\partial y} \frac{\partial^{s+3} v}{\partial x^{s} \partial y^{3}}\left(x_{T}, y\right) d y= & -\left.U\left(x_{T} \pm h_{1, T}, y\right) \frac{\partial^{s+3} v}{\partial x^{s} \partial y^{3}}\left(x_{T}, y\right)\right|_{y_{T}-h_{2, T}} ^{y_{T}+h_{2, T}} \\
& +\int_{l_{i}} U \frac{\partial^{s+4} v}{\partial x^{s} \partial y^{4}}\left(x_{T}, y\right) d y=0
\end{aligned}
$$

By analogy, it is easy to get the equality:

$$
\int_{l_{i}} \frac{\partial}{\partial x}\left[\frac{\partial^{2} U}{\partial y^{2}} \frac{\partial^{s+2} v}{\partial x^{s} \partial y^{2}}\left(x_{T}, y\right)\right] d y=\int_{l_{i}} \frac{\partial^{3} U}{\partial x \partial y^{2}} \frac{\partial^{s+2} v}{\partial x^{s} \partial y^{2}}\left(x_{T}, y\right) d y=0,
$$

having in mind the definition of $U$.

For $s=2 ; 4$ and using the Green's formula we get:

$$
\begin{aligned}
\int_{T} \frac{\partial^{2} U}{\partial y^{2}} \frac{\partial^{s+2} v}{\partial x^{s} \partial y^{2}}\left(x_{T}, y\right) d x d y & =\left(\int_{l_{3}}-\int_{l_{1}}\right) \frac{\partial U}{\partial y} \frac{\partial^{s+2} v}{\partial x^{s} \partial y^{2}}\left(x_{T}, y\right) d x \\
-\int_{T} \frac{\partial U}{\partial y} \frac{\partial^{s+3} v}{\partial x^{s} \partial y^{3}}\left(x_{T}, y\right) d x d y & =\left(\int_{l_{3}}-\int_{l_{1}}\right) U \frac{\partial^{s+3} v}{\partial x^{s} \partial y^{3}}\left(x_{T}, y\right) d x \\
+\int_{T} U \frac{\partial^{s+4} v}{\partial x^{s} \partial y^{4}}\left(x_{T}, y\right) d x d y & =0 .
\end{aligned}
$$

Then, in view of (15) and (16), applying (8) of Lemma 1 for the function $\frac{\partial^{2} U}{\partial y^{2}} \frac{\partial^{s+2} v}{\partial x^{s} \partial y^{2}}\left(x_{T}, y\right)$ with $s=1$ and $s=3$ respectively, we obtain:

$$
\begin{aligned}
\int_{T}\left(x-x_{T}\right) \frac{\partial^{2} U}{\partial y^{2}} \frac{\partial^{3} v}{\partial x \partial y^{2}}\left(x_{T}, y\right) d x & d y \\
& =\frac{1}{90} \int_{T} E^{3}(x) \frac{\partial^{7} u}{\partial x^{5} \partial y^{2}} \frac{\partial^{3} v}{\partial x \partial y^{2}}\left(x_{T}, y\right) d x d y
\end{aligned}
$$




$$
\begin{gathered}
\int_{T} \frac{\left(x-x_{T}\right)^{3}}{3 !} \frac{\partial^{2} U}{\partial y^{2}} \frac{\partial^{5} v}{\partial x^{3} \partial y^{2}}\left(x_{T}, y\right) d x d y=\frac{1}{420.3 !} \int_{T} E^{4}(x) \frac{\partial^{7} u}{\partial x^{5} \partial y^{2}} \frac{\partial^{5} v}{\partial x^{3} \partial y^{2}}\left(x_{T}, y\right) d x d y \\
+\frac{h_{1, T}^{2}}{210.3 !} \int_{T} E^{3}(x) \frac{\partial^{7} u}{\partial x^{5} \partial y^{2}} \frac{\partial^{5} v}{\partial x^{3} \partial y^{2}}\left(x_{T}, y\right) d x d y
\end{gathered}
$$

Similarly, we get:

$$
\begin{aligned}
\int_{T} \frac{\left(x-x_{T}\right)^{2}}{2 !} \frac{\partial^{2} U}{\partial y^{2}} \frac{\partial^{4} v}{\partial x^{2} \partial y^{2}}\left(x_{T}, y\right) d x d y & =-\frac{1}{1260.2 !} \int_{T} E^{4}(x) \frac{\partial^{8} u}{\partial x^{6} \partial y^{2}} \frac{\partial^{4} v}{\partial x^{2} \partial y^{2}}\left(x_{T}, y\right) d x d y \\
\int_{T} \frac{\left(x-x_{T}\right)^{4}}{4 !} \frac{\partial^{2} U}{\partial y^{2}} \frac{\partial^{6} v}{\partial x^{4} \partial y^{2}}(x, y) d x d y & =-\frac{1}{4725.4 !} \int_{T} E^{5}(x) \frac{\partial^{8} u}{\partial x^{6} \partial y^{2}} \frac{\partial^{6} v}{\partial x^{4} \partial y^{2}}(x, y) d x d y \\
& -\frac{h_{1, T}^{2}}{1890.4 !} \int_{T} E^{4}(x) \frac{\partial^{8} u}{\partial x^{6} \partial y^{2}} \frac{\partial^{6} v}{\partial x^{4} \partial y^{2}}(x, y) d x d y,
\end{aligned}
$$

having in mind (15)-(17) and applying (9) of Lemma 1 for the same function with $s=2$ and $s=4$, respectively.

It is possible to reduce the requirement for high smoothness without loss of accuracy. Indeed, using the identity

$$
\left[E^{k}(x)\right]^{\prime}=k E^{k-1}(x) \cdot\left(x-x_{T}\right), \quad k=4 ; 5,
$$

the last two equalities may be written in the form

$$
\begin{aligned}
& \int_{T} \frac{\left(x-x_{T}\right)^{2}}{2 !} \frac{\partial^{2} U}{\partial y^{2}} \frac{\partial^{4} v}{\partial x^{2} \partial y^{2}}\left(x_{T}, y\right) d x d y=\frac{1}{315.2 !} \int_{T} E^{3}(x)\left(x-x_{T}\right) \frac{\partial^{7} u}{\partial x^{5} \partial y^{2}} \frac{\partial^{4} v}{\partial x^{2} \partial y^{2}}\left(x_{T}, y\right) d x d y \\
& \int_{T} \frac{\left(x-x_{T}\right)^{4}}{4 !} \frac{\partial^{2} U}{\partial y^{2}} \frac{\partial^{6} v}{\partial x^{4} \partial y^{2}}(x, y) d x d y \\
&=\frac{1}{945.4 !} \int_{T} E^{4}(x)\left(x-x_{T}\right) \frac{\partial^{7} u}{\partial x^{5} \partial y^{2}} \frac{\partial^{6} v}{\partial x^{4} \partial y^{2}}(x, y) d x d y \\
& \quad+\frac{h_{1, T}^{2}}{1890.3 !} \int_{T} E^{3}(x)\left(x-x_{T}\right) \frac{\partial^{7} u}{\partial x^{5} \partial y^{2}} \frac{\partial^{6} v}{\partial x^{4} \partial y^{2}}(x, y) d x d y
\end{aligned}
$$

The right-hand side of (18), (19) and (20) can be transformed for $v \in V_{h}$ and $T \in \tau_{h}$ by the identity

$$
\frac{\partial^{s+2} v}{\partial x^{s} \partial y^{2}}\left(x_{T}, y\right)=\sum_{i=s}^{4} \frac{\left(x_{T}-x\right)^{i-s}}{(i-s) !} \frac{\partial^{i+2} v}{\partial x^{i} \partial y^{2}}(x, y), \quad s=1,2,3 ; \quad(x, y) \in T .
$$


From (14), (18)-(21), using the inverse inequality [5], we obtain

$$
\int_{T} \frac{\partial^{2} U}{\partial y^{2}} \frac{\partial^{2} v}{\partial y^{2}} d x d y \leq C h^{5}\|u\|_{7, T}\|v\|_{2, T},
$$

consequently,

$$
\int_{\Omega} \frac{\partial^{2} U}{\partial y^{2}} \frac{\partial^{2} v}{\partial y^{2}} d x d y \leq C h^{5}\|u\|_{7, \Omega}\|v\|_{2, \Omega}
$$

and by analogy

$$
\int_{\Omega} \frac{\partial^{2} U}{\partial x^{2}} \frac{\partial^{2} v}{\partial x^{2}} d x d y \leq C h^{5}\|u\|_{7, \Omega}\|v\|_{2, \Omega} .
$$

Step 2. Here we consider the mixed term of the $a$-form. Let's consider that the following equalities hold:

$$
\begin{aligned}
\sum_{T \in \tau_{h}} \int_{T} \frac{\partial^{2} U}{\partial x \partial y} \frac{\partial^{2} v}{\partial x \partial y} d x d y & =\sum_{T \in \tau_{h}}\left(\int_{l_{3}}-\int_{l_{1}}\right) \frac{\partial^{2} U}{\partial x \partial y} \frac{\partial v}{\partial x} d x \\
-\sum_{T \in \tau_{h}} \int_{T} \frac{\partial^{3} U}{\partial x \partial y^{2}} \frac{\partial v}{\partial x} d x d y & =-\sum_{T \in \tau_{h}}\left(\int_{l_{2}}-\int_{l_{4}}\right) \frac{\partial^{3} U}{\partial x \partial y^{2}} v d x \\
+\sum_{T \in \tau_{h}} \int_{T} \frac{\partial^{4} U}{\partial x^{2} \partial y^{2}} v d x d y & =\sum_{T \in \tau_{h}} \int_{T} \frac{\partial^{4} U}{\partial x^{2} \partial y^{2}} v d x d y
\end{aligned}
$$

because the functions $\frac{\partial^{2} U}{\partial x \partial y} \frac{\partial v}{\partial y}$ and $\frac{\partial^{3} U}{\partial x \partial y^{2}} v$ are continuous on the edges, parallel to the $x$ and $y$-axes, respectively. For the line integrals we integrate over the common side of each pair of two adjacent elements with the same integrands, but in opposite direction. For integrals over any parts of $\Gamma$ the function $v$ vanishes.

Consider the following presentation for any $v \in V_{h}$ :

$$
v(x, y)=\sum_{i, j=0}^{4} \frac{1}{i ! j !}\left(x-x_{T}\right)^{i}\left(y-y_{T}\right)^{j} \frac{\partial^{i+j} v}{\partial x^{i} \partial y^{j}}\left(x_{T}, y_{T}\right) \quad \forall(x, y) \in T .
$$

Combining this equality with (24) we get:

$$
\int_{T} \frac{\partial^{4} U}{\partial x^{2} \partial y^{2}} v d x d y=\sum_{i, j=0}^{4} \int_{T} \frac{\left(x-x_{T}\right)^{i}\left(y-y_{T}\right)^{j}}{i ! j !} \frac{\partial^{4} U}{\partial x^{2} \partial y^{2}} \frac{\partial^{i+j} v}{\partial x^{i} \partial y^{j}}\left(x_{T}, y_{T}\right) d x d y
$$

We shall estimate each term in (25) using the relations of Lemma 2.

First, from $\left(R_{1}\right)$ for $i, j=0,1,2$ it follows that

$$
\int_{T} \frac{\left(x-x_{T}\right)^{i}\left(y-y_{T}\right)^{j}}{i ! j !} \frac{\partial^{4} U}{\partial x^{2} \partial y^{2}} \frac{\partial^{i+j} v}{\partial x^{i} \partial y^{j}}\left(x_{T}, y_{T}\right) d x d y=0 .
$$

Next, the relation $\left(R_{2}\right)$ for $i ; j=0,1,2$ respectively gives

$$
\int_{T} \frac{\left(x-x_{T}\right)^{3}\left(y-y_{T}\right)^{j}}{3 ! j !} \frac{\partial^{4} U}{\partial x^{2} \partial y^{2}} \frac{\partial^{3+j} v}{\partial x^{3} \partial y^{j}}\left(x_{T}, y_{T}\right) d x d y
$$




$$
\begin{aligned}
= & \frac{1}{15.3 ! j !} \int_{T} E^{3}(x)\left(y-y_{T}\right)^{j} \frac{\partial^{7} u}{\partial x^{5} \partial y^{2}} \frac{\partial^{3+j} v}{\partial x^{3} \partial y^{j}}\left(x_{T}, y_{T}\right) d x d y \\
& \int_{T} \frac{\left(x-x_{T}\right)^{i}\left(y-y_{T}\right)^{3}}{i ! 3 !} \frac{\partial^{4} U}{\partial x^{2} \partial y^{2}} \frac{\partial^{i+3} v}{\partial x^{i} \partial y^{3}}\left(x_{T}, y_{T}\right) d x d y \\
= & \frac{1}{15 . i ! 3 !} \int_{T}\left(x-x_{T}\right)^{i} F^{3}(y) \frac{\partial^{7} u}{\partial x^{2} \partial y^{5}} \frac{\partial^{i+3} v}{\partial x^{i} \partial y^{3}}\left(x_{T}, y_{T}\right) d x d y .
\end{aligned}
$$

By analogy with (27), using $\left(R_{3}\right)$ for $i ; j=0,1,2$, we obtain the next two equalities:

$$
\begin{gathered}
\int_{T} \frac{\left(x-x_{T}\right)^{4}\left(y-y_{T}\right)^{j}}{4 ! j !} \frac{\partial^{4} U}{\partial x^{2} \partial y^{2}} \frac{\partial^{4+j} v}{\partial x^{4} \partial y^{j}}\left(x_{T}, y_{T}\right) d x d y \\
=-\frac{1}{105.4 ! j !} \int_{T} E^{4}(x)\left(y-y_{T}\right)^{j} \frac{\partial^{8} u}{\partial x^{6} \partial y^{2}} \frac{\partial^{4+j} v}{\partial x^{4} \partial y^{j}}\left(x_{T}, y_{T}\right) d x d y \\
=\frac{1}{105.3 ! j !} \int_{T} E^{3}(x)\left(x-x_{T}\right)\left(y-y_{T}\right)^{j} \frac{\partial^{7} u}{\partial x^{5} \partial y^{2}} \frac{\partial^{4+j} v}{\partial x^{4} \partial y^{j}}\left(x_{T}, y_{T}\right) d x d y \\
=-\frac{1}{105 . i ! 4 !} \int_{T}\left(x-x_{T}\right)^{i} F^{4}(y) \frac{\partial^{8} u}{\partial x^{2} \partial y^{6}} \frac{\partial^{i+4} v}{\partial x^{i} \partial y^{4}}\left(x_{T}, y_{T}\right) d x d y \\
=\frac{1}{105 . i ! 3 !} \int_{T}\left(x-x_{T}\right)^{i}\left(y-y_{T}\right) F^{3}(y) \frac{\partial^{7} u}{\partial x^{2} \partial y^{5}} \frac{\partial^{i+4} v}{\partial x^{i} \partial y^{4}}\left(x_{T}, y_{T}\right) d x d y .
\end{gathered}
$$

From $\left(R_{4}\right)$ it follows that

$$
\begin{aligned}
& \int_{T} \frac{\left(x-x_{T}\right)^{3}\left(y-y_{T}\right)^{3}}{3 ! 3 !} \frac{\partial^{4} U}{\partial x^{2} \partial y^{2}} \frac{\partial^{6} v}{\partial x^{3} \partial y^{3}}\left(x_{T}, y_{T}\right) d x d y \\
= & \frac{1}{15.3 ! .3 !} \int_{T} E^{3}(x)\left(y-y_{T}\right)^{3} \frac{\partial^{7} u}{\partial x^{5} \partial y^{2}} \frac{\partial^{6} v}{\partial x^{3} \partial y^{3}}\left(x_{T}, y_{T}\right) d x d y \\
+ & \frac{h_{1, T}^{2}}{25.3 ! 3 !} \int_{T}\left(x-x_{T}\right) F^{3}(y) \frac{\partial^{7} u}{\partial x^{2} \partial y^{5}} \frac{\partial^{6} v}{\partial x^{3} \partial y^{3}}\left(x_{T}, y_{T}\right) d x d y .
\end{aligned}
$$

Introducing the equalities for sufficiently smooth function $U$

$$
\begin{aligned}
& \int_{T} E^{4}(x) \frac{\partial^{6} U}{\partial x^{6}} d x d y=-4 \int_{T}\left(x-x_{T}\right) E^{3}(x) \frac{\partial^{5} U}{\partial x^{5}} d x d y \\
& \int_{T} F^{4}(y) \frac{\partial^{6} U}{\partial y^{6}} d x d y=-4 \int_{T}\left(y-y_{T}\right) F^{3}(y) \frac{\partial^{5} U}{\partial y^{5}} d x d y
\end{aligned}
$$

from the relation $\left(R_{4}\right)$ we get

$$
\int_{T} \frac{\left(x-x_{T}\right)^{4}\left(y-y_{T}\right)^{3}}{4 ! 3 !} \frac{\partial^{4} U}{\partial x^{2} \partial y^{2}} \frac{\partial^{7} v}{\partial x^{4} \partial y^{3}}\left(x_{T}, y_{T}\right) d x d y
$$




$$
\begin{gathered}
=\frac{1}{105.3 ! .3 !} \int_{T} E^{3}(x)\left(x-x_{T}\right)\left(y-y_{T}\right)^{3} \frac{\partial^{7} u}{\partial x^{5} \partial y^{2}} \frac{\partial^{7} v}{\partial x^{4} \partial y^{3}}\left(x_{T}, y_{T}\right) d x d y \\
+\frac{2 h_{1, T}^{2}}{35.4 ! 3 !} \int_{T}\left(x-x_{T}\right)^{2} F^{3}(y) \frac{\partial^{7} u}{\partial x^{2} \partial y^{5}} \frac{\partial^{7} v}{\partial x^{4} \partial y^{3}}\left(x_{T}, y_{T}\right) d x d y \\
\quad-\frac{h_{1, T}^{4}}{175.4 ! 3 !} \int_{T} F^{3}(y) \frac{\partial^{7} u}{\partial x^{2} \partial y^{5}} \frac{\partial^{7} v}{\partial x^{4} \partial y^{3}}\left(x_{T}, y_{T}\right) d x d y
\end{gathered}
$$

Similarly,

$$
\begin{gathered}
\int_{T} \frac{\left(x-x_{T}\right)^{3}\left(y-y_{T}\right)^{4}}{3 ! 4 !} \frac{\partial^{4} U}{\partial x^{2} \partial y^{2}} \frac{\partial^{7} v}{\partial x^{3} \partial y^{4}}\left(x_{T}, y_{T}\right) d x d y \\
=\frac{1}{105.3 ! .3 !} \int_{T}\left(x-x_{T}\right)^{3}\left(y-y_{T}\right) F^{3}(y) \frac{\partial^{7} u}{\partial x^{2} \partial y^{5}} \frac{\partial^{7} v}{\partial x^{3} \partial y^{4}}\left(x_{T}, y_{T}\right) d x d y \\
+\frac{2 h_{2, T}^{2}}{35.3 ! 4 !} \int_{T} E^{3}(x)\left(y-y_{T}\right)^{2} \frac{\partial^{7} u}{\partial x^{5} \partial y^{2}} \frac{\partial^{7} v}{\partial x^{3} \partial y^{4}}\left(x_{T}, y_{T}\right) d x d y \\
-\frac{h_{2, T}^{4}}{175.3 ! 4 !} \int_{T} E^{3}(x) \frac{\partial^{7} u}{\partial x^{5} \partial y^{2}} \frac{\partial^{7} v}{\partial x^{3} \partial y^{4}}\left(x_{T}, y_{T}\right) d x d y
\end{gathered}
$$

By analogy

$$
\begin{gathered}
\int_{T} \frac{\left(x-x_{T}\right)^{4}\left(y-y_{T}\right)^{4}}{4 ! 4 !} \frac{\partial^{4} U}{\partial x^{2} \partial y^{2}} \frac{\partial^{8} v}{\partial x^{4} \partial y^{4}} d x d y \\
=\frac{1}{105.4 ! .3 !} \int_{T} E^{3}(x)\left(x-x_{T}\right)\left(y-y_{T}\right)^{4} \frac{\partial^{7} u}{\partial x^{5} \partial y^{2}} \frac{\partial^{8} v}{\partial x^{4} \partial y^{4}} d x d y \\
+\frac{2 h_{1, T}^{2}}{245.4 ! 4 !} \int_{T}\left(x-x_{T}\right)^{2}\left(y-y_{T}\right) F^{3}(y) \frac{\partial^{7} u}{\partial x^{2} \partial y^{5}} \frac{\partial^{8} v}{\partial x^{4} \partial y^{4}} d x d y \\
-\frac{h_{1, T}^{4}}{1225.4 ! 4 !} \int_{T}\left(y-y_{T}\right) F^{3}(y) \frac{\partial^{7} u}{\partial x^{2} \partial y^{5}} \frac{\partial^{8} v}{\partial x^{4} \partial y^{4}} d x d y .
\end{gathered}
$$

In the right-hand sides of (27)-(31) we apply the equality

$$
\frac{\partial^{i+j} v}{\partial x^{i} \partial y^{j}}\left(x_{T}, y_{T}\right)=\sum_{i_{0}=i, j_{0}=j}^{4} \frac{\left(x-x_{T}\right)^{i_{0}-i}\left(y-y_{T}\right)^{j_{0}-j}}{\left(i_{0}-i\right) !\left(j_{0}-j\right) !} \frac{\partial^{i_{0}+j_{0}} v}{\partial x^{i_{0}} \partial y^{j_{0}}}(x, y),
$$

for corresponding values of $i$ and $j$.

Thus, from (26)-(32) and using the inverse inequality, we obtain

$$
\int_{T} \frac{\partial^{2} U}{\partial x \partial y} \frac{\partial^{2} v}{\partial x \partial y} d x d y \leq C h_{T}^{5}\|u\|_{7, T}\|v\|_{2, T}
$$

and consequently,

$$
\int_{\Omega} \frac{\partial^{2} U}{\partial x \partial y} \frac{\partial^{2} v}{\partial x \partial y} d x d y \leq C h^{5}\|u\|_{7, \Omega}\|v\|_{2, \Omega} .
$$

Accordingly, the three terms of $a$-form are estimated. Hence (22), (23) and (33) prove the theorem. 
As a consequence of this theorem the following ultraconvergence result holds:

Theorem 2. Let the conditions of Theorem 1 be fulfilled. Then

$$
\left\|i_{h} u-P_{h} u\right\|_{2, \Omega} \leq C h^{5}\|u\|_{7, \Omega}
$$

Proof. It follows that $(\alpha=$ const):

$$
\begin{aligned}
\alpha\left\|i_{h} u-P_{h} u\right\|_{2, \Omega}^{2} & \leq a\left(i_{h} u-P_{h} u, i_{h} u-P_{h} u\right) & & \text { (V-ellipticity) } \\
& =a\left(i_{h} u-u, i_{h} u-P_{h} u\right) & & \text { (elliptic projector) } \\
& \leq C\left\|i_{h} u-u\right\|_{2, \Omega}\left\|i_{h} u-P_{h} u\right\|_{2, \Omega} & & \text { (continuity) } \\
& \leq C h^{5}\|u\|_{7, \Omega}\left\|i_{h} u-P_{h} u\right\|_{2, \Omega} & & \text { (from Theorem } 1) .
\end{aligned}
$$

\section{HIGH INTERPOLATION OPERATOR $\mathrm{I}_{2 \mathrm{H}}-$ CONSTRUCTION AND ESTIMATIONS}

First, let us consider the one-dimensional case. We keep the same notation for onedimensional FE partition, namely $\tau_{h}=\left\{\left[p_{i-1}, p_{i}\right], i=1, \ldots, n\right\}$. Without loss of generality one can suppose that $n$ is an even natural number. Then the elements of $\tau_{h}$ are combined by pairs of adjacent elements. A new FE partition is defined by

$$
\widetilde{\tau}_{2 h}=\left\{\left[p_{2 k-2}, p_{2 k-1}\right] \cup\left[p_{2 k-1}, p_{2 k}\right], k=1, \ldots, \frac{n}{2}\right\} .
$$

The interpolation operator $I_{2 h}$ is characterized by the following conditions for every finite element belonging to $\widetilde{\tau}_{2 h}$ and every smooth function $v$ :

$$
\begin{gathered}
I_{2 h} v\left(p_{j}\right)=v\left(p_{j}\right), \quad j=2 k-2,2 k-1,2 k ; \\
{\left[I_{2 h} v\right]^{\prime}\left(p_{j}\right)=v^{\prime}\left(p_{j}\right), \quad j=2 k-2,2 k-1,2 k ;} \\
\int_{p_{j}}^{p_{j+1}} I_{2 h} v(x) d x=\int_{p_{j}}^{p_{j+1}} v(x) d x, \quad j=2 k-2,2 k-1 .
\end{gathered}
$$

For instance, it is possible to obtain the following basic functions defined on a 
reference element, i.e. for $t \in[-1,0] \cup[0,1]$ :

$$
\begin{aligned}
\psi_{1 ; 7}(t) & =\frac{1}{16} t^{2}(1-t)^{2}\left( \pm 72 t^{3}+95 t^{2} \mp 14 t-33\right), \\
\psi_{2 ; 8}(t) & =\frac{1}{16} t^{2}(1-t)^{2}\left(8 t^{3} \pm 9 t^{2}-2 t \mp 33\right), \\
\psi_{3 ; 6}(t) & =\frac{1}{16} t^{2}(1-t)^{2}(1+t)^{2}(35 \mp 64 t), \\
\psi_{4}(t) & =(1-t)^{2}(1+t)^{2}\left(1-7 t^{2}\right), \\
\psi_{5}(t) & =t(1-t)^{2}(1+t)^{2}(1-2 t)(1+2 t) .
\end{aligned}
$$

By affine transformation

$$
t=\frac{x-p_{2 k-1}}{h}
$$

where

$$
h= \begin{cases}p_{2 k}-p_{2 k-1}, & x \in\left[p_{2 k-1}, p_{2 k}\right], \\ p_{2 k-1}-p_{2 k-2}, & x \in\left[p_{2 k-2}, p_{2 k-1}\right],\end{cases}
$$

it is not difficult to obtain the basic functions $\psi_{j}(x), j=1, \ldots, 8$ for any element $\left[p_{2 k-2}, p_{2 k-1}\right] \cup\left[p_{2 k-1}, p_{2 k}\right] \in \widetilde{\tau}_{2 h}$.

The set of degrees of freedom is $v\left(p_{j}\right), h v^{\prime}\left(p_{j}\right), j=2 k-2,2 k-1,2 k$ and $\frac{1}{h} \int_{p_{j}}^{p_{j+1}} v(x) d x, j=2 k-2,2 k-1$.

For 2-dimensional case the basic functions are defined as a product of two onedimensional basic functions, i.e. $\psi_{i, j}(x, y)=\psi_{i}(x) \psi_{j}(y), i, j=1, \ldots, 8$. Consequently, the degrees of freedom are: $(i)$ the values of $v, \frac{\partial v}{\partial x}, \frac{\partial v}{\partial y}$ and $\frac{\partial^{2} v}{\partial x \partial y}$ on the vertices of the four subrectangles $T_{s} \in \tau_{h}, s=1, \ldots, 4$ such that $\bigcup_{s=1}^{4} T_{s} \in \widetilde{\tau}_{2 h} ;(i i)$ the integral values of $v$ and $\frac{\partial v}{\partial \nu}$ on the edges $l_{j}$ of $T_{s} \in \tau_{h}, s=1, \ldots, 4 ;(i i i)$ the integral values of $v$ on the four subrectangles $T_{s}, s=1, \ldots, 4$ multiplied or divided by the corresponding sizes characterizing the mesh of $\tau_{h}$.

In this way the operator $I_{2 h}$ is defined on the mesh $\widetilde{\tau}_{2 h}$ of size $2 h$, obtained as a result of arranging in groups of adjacent elements $T_{s} \in \tau_{h}, s=1, \ldots, 4$. Let $\widetilde{V}_{2 h} \subset V$ be finite element spaces associated with $\widetilde{\tau}_{2 h}$. Then $\widetilde{V}_{2 h}$ consists of polynomials of degree 7 at most in each variable.

By constructing $I_{2 h}$, the following properties are valid:

$$
\begin{gathered}
I_{2 h} \circ i_{h}=I_{2 h}, \\
\left\|I_{2 h} v\right\|_{r, \Omega} \leq C\|v\|_{r, \Omega} \quad \forall v \in V_{h}, r=0,1,2,
\end{gathered}
$$

because the interpolation operator $I_{2 h}: V_{h} \rightarrow \widetilde{V}_{2 h}$ is bounded. 
Finally, having in mind that the interpolation polynomial $I_{2 h} v$ is of degree seven, it follows

$$
\left\|I_{2 h} v-v\right\|_{2, \Omega} \leq C h^{6}\|v\|_{7, \Omega} .
$$

The next theorem contains the main estimation:

Theorem 3. Let $u \in H^{7}(\Omega)$. The following estimate holds:

$$
\left\|I_{2 h} \circ P_{h} u-u\right\|_{2, \Omega} \leq C h^{5}\|u\|_{7, \Omega} .
$$

Proof. Applying (35), brings to

$$
I_{2 h} \circ P_{h} u-u=I_{2 h} \circ\left(P_{h} u-i_{h} u\right)+\left(I_{2 h} u-u\right) .
$$

From (36) we have

$$
\left\|I_{2 h} \circ P_{h} u-u\right\|_{2, \Omega} \leq\left\|P_{h} u-i_{h} u\right\|_{2, \Omega}+\left\|I_{2 h} u-u\right\|_{2, \Omega} .
$$

Using (34) and (37), we complete the proof.

\section{ULTRACONVERGENCE FOR BIHARMONIC EIGENVALUE PROBLEM}

Here we present a direct application of the result obtained in the previous section. Consider the following fourth-order elliptic eigenvalue problem: find $\lambda \in \mathbf{R}, u(x, y) \in$ $V, u \neq 0$, such that

$$
a(u, v)=\lambda(u, v), \quad \forall v \in V .
$$

Introduce an approximate eigenvalue problem, which corresponds to (39): find $\lambda_{h} \in \mathbf{R}, u_{h}(x, y) \in V_{h}, u_{h} \neq 0$, such that

$$
a\left(u_{h}, v\right)=\lambda_{h}\left(u_{h}, v\right), \quad \forall v \in V_{h} .
$$

The assumption here is that the FE space $V_{h}$ uses polynomials of degree four. A more general case is discussed in [2]. That being the case, it is well known (see [4, 8]) that the rate of convergence of FE approximation to the eigenvalues and eigenfunctions is given by the following estimates:

$$
\begin{gathered}
\left|\lambda-\lambda_{h}\right| \leq C(\lambda) h^{6}\|u\|_{5, \Omega}^{2}, \\
\left\|u-u_{h}\right\|_{2, \Omega} \leq C(\lambda) h^{3}\|u\|_{5, \Omega} .
\end{gathered}
$$

The solutions of (39) and (40) are related to the Rayleigh quotient

$$
\lambda=\frac{a(u, u)}{(u, u)} \text { and } \lambda_{h}=\frac{a\left(u_{h}, u_{h}\right)}{\left(u_{h}, u_{h}\right)} .
$$


First, let us estimate the difference $u_{h}-P_{h} u$ in $H^{2}$-norm of higher order of accuracy as compared to the estimates (4) and (42). This special feature of both functions is called to satisfy a superclose property (cf. [12]).

An important result related to the ultraconvergence patch-recovery for approximate eigenpairs is presented by the following lemma:

Lemma 3. Let the eigenfunction $u(x, y) \in H^{5}(\Omega) \cap V$ and let $u_{h}$ be the corresponding FE approximation obtained by (40). Then

$$
\left\|u_{h}-P_{h} u\right\|_{2, \Omega} \leq C h^{6}\|u\|_{5, \Omega}
$$

Proof. What is obtained from the ellipticity on the FE space is $\left(\rho_{1}=\right.$ const):

$$
\rho_{1}\left\|u_{h}-P_{h} u\right\|_{2, \Omega}^{2} \leq a\left(u_{h}-P_{h} u, u_{h}-P_{h} u\right) .
$$

Let us denote $u_{h}-P_{h} u=z_{h} \in V_{h}$. Using the orthogonal property of $P_{h}$, it follows that

$$
\begin{aligned}
\rho_{1}\left\|z_{h}\right\|_{2, \Omega}^{2} & \leq \lambda_{h}\left(u_{h}, z_{h}\right)-a\left(P_{h} u, z_{h}\right) \\
& =\left(\lambda_{h}-\lambda\right)\left(u_{h}, z_{h}\right)+\lambda\left(u_{h}, z_{h}\right)-a\left(u, z_{h}\right) \\
& =\left(\lambda_{h}-\lambda\right)\left(u_{h}, z_{h}\right)+\lambda\left(u_{h}-u, z_{h}\right)+\lambda\left(u, z_{h}\right)-a\left(u, z_{h}\right) \\
& =\left(\lambda_{h}-\lambda\right)\left(u_{h}, z_{h}\right)+\lambda\left(u_{h}-u, z_{h}\right) \\
& \leq\left|\lambda-\lambda_{h}\right|\left\|u_{h}\right\|_{0, \Omega}\left\|z_{h}\right\|_{2, \Omega}+\lambda\left\|u_{h}-u\right\|_{-1, \Omega}\left\|z_{h}\right\|_{1, \Omega} .
\end{aligned}
$$

Note that in the last step we use the duality in negative norms. The estimate (43) follows from (41) and the inequality [9]

$$
\left\|u_{h}-u\right\|_{-1, \Omega} \leq C h^{6}\|u\|_{5, \Omega} .
$$

In order to prove our main result concerning the ultraconvergence patch-recovery to the approximate eigenpairs we need the following lemma:

Lemma 4. Let $(\lambda, u)$ be any eigenpair obtained by (39). Then for every $w \in V$ and $w \neq 0$, the following inequality holds:

$$
\left|\frac{a(w, w)}{(w, w)}-\lambda\right| \leq C \frac{\|w-u\|_{2, \Omega}^{2}}{(w, w)} .
$$


Proof. Denote $w-u=\varphi \in V \equiv H_{0}^{2}(\Omega)$, consequently $w=u+\varphi$. Hence the estimate (44) is equivalent to

$$
|a(u+\varphi, u+\varphi)-\lambda(u+\varphi, u+\varphi)| \leq C\|\varphi\|_{2, \Omega}^{2} \quad \forall v \in V .
$$

We transform the left-hand side of this inequality:

$$
\begin{aligned}
\mid a(u, u)+ & 2 a(u, \varphi)+a(\varphi, \varphi)-\lambda(u, u)-2 \lambda(u, \varphi)-\lambda(\varphi, \varphi) \mid \\
& =|a(u, u)+a(\varphi, \varphi)-\lambda(u, u)-\lambda(\varphi, \varphi)| .
\end{aligned}
$$

Giving an account of $a(u, u)=\lambda(u, u)$, it follows from the continuity that

$$
\begin{aligned}
|a(u+\varphi, u+\varphi)-\lambda(u+\varphi, u+\varphi)| & =|a(\varphi, \varphi)-\lambda(\varphi, \varphi)| \\
& \leq C_{1}\|\varphi\|_{2, \Omega}^{2}+\lambda\|\varphi\|_{0, \Omega}^{2} \\
& \leq C\|\varphi\|_{2, \Omega}^{2},
\end{aligned}
$$

which proves the lemma.

The main result of this section is contained in the next theorem:

Theorem 4. Let $(\lambda, u)$ be an exact eigenpair and $\left(\lambda_{h}, u_{h}\right)$ be its FE approximation. Assume that the conditions of Theorem 3 and Lemma 3 are fulfilled such that the ultraconvergence estimations (38) and (43) hold. Then

$$
\begin{gathered}
\left\|I_{2 h} u_{h}-u\right\|_{2, \Omega} \leq C h^{5}\|u\|_{7, \Omega}, \\
\left|\frac{a\left(I_{2 h} u_{h}, I_{2 h} u_{h}\right)}{\left(I_{2 h} u_{h}, I_{2 h} u_{h}\right)}-\lambda\right| \leq C h^{10}\|u\|_{7, \Omega} .
\end{gathered}
$$

Proof. The estimate (45) can be proved by Theorem 3 and Lemma 3:

$$
\begin{aligned}
\left\|I_{2 h} u_{h}-u\right\|_{2, \Omega} & \leq\left\|I_{2 h} u_{h}-I_{2 h} P_{h} u\right\|_{2, \Omega}+\left\|I_{2 h} P_{h} u-u\right\|_{2, \Omega} \\
& \leq\left\|I_{2 h}\right\|\left\|u_{h}-P_{h} u\right\|_{2, \Omega}+\left\|I_{2 h} P_{h} u-u\right\|_{2, \Omega} .
\end{aligned}
$$

The interpolation operator $I_{2 h}: V_{h} \rightarrow \widetilde{V}_{2 h}$ is compact. So

$$
\left\|I_{2 h}\right\|=\sup _{v_{h} \in V_{h}} \frac{\left\|I_{2 h} v_{h}\right\|_{2, \Omega}}{\left\|v_{h}\right\|_{2, \Omega}} \leq \text { const. }
$$

Therefore

$$
\left\|I_{2 h} u_{h}-u\right\|_{2, \Omega} \leq C_{1} h^{6}\|u\|_{5, \Omega}+C_{2} h^{5}\|u\|_{7, \Omega} .
$$


In order to prove the inequality (46) Lemma 4 and (45) should be used:

$$
\begin{aligned}
\left|\frac{a\left(I_{2 h} u_{h}, I_{2 h} u_{h}\right)}{\left(I_{2 h} u_{h}, I_{2 h} u_{h}\right)}-\lambda\right| & \leq C \frac{\left\|I_{2 h} u_{h}-u\right\|_{2, \Omega}^{2}}{\left\|I_{2 h} u_{h}\right\|_{0, \Omega}^{2}} \\
& \leq C h^{10}\|u\|_{7, \Omega}^{2} .
\end{aligned}
$$

\section{NUMERICAL RESULTS}

Consider a simple model problem of a thin bar of length $l$ which is simply supported at its endpoints. The flexural rigidity and the density of the rod are units. Accordingly, we solve the following differential system:

$$
\begin{gathered}
u^{I V}=\lambda u, \quad x \in(0, l), \\
u(0)=u^{\prime}(0)=0, \\
u(l)=u^{\prime}(l)=0 .
\end{gathered}
$$

The exact eigenvalues $\lambda_{j}, j=1,2, \ldots$ satisfy the equation $\left(k_{j}=\sqrt[4]{\lambda_{j}}\right)$ :

$$
\cos k_{j} l \cosh k_{j} l=1
$$

and the exact eigenfunction are

$$
\begin{aligned}
u_{j}(x)=C\left[( \operatorname { s i n h } k _ { j } l - \operatorname { s i n } k _ { j } l ) \left(\cosh k_{j} x\right.\right. & \left.-\cos k_{j} x\right) \\
& \left.-\left(\cosh k_{j} l-\cos k_{j} l\right)\left(\sinh k_{j} x-\sin k_{j} x\right)\right] .
\end{aligned}
$$

When $l=1$, the first three exact eigenvalues are:

$$
\lambda_{1}=500.563901740433, \quad \lambda_{2}=3803.53708049787, \quad \lambda_{3}=14617.6301311223 .
$$

Though one-dimensional case is under consideration here, it still presents a relevant illustration of rectangular FE theory.

In Table 1 and Table 2 the efficiency of patch-recovery technique is illustrated. $N_{e}$ denotes the number of elements in partition $\tau_{h}$.

Let us introduce

$$
\tilde{\lambda}_{h, j}=\frac{a\left(I_{2 h} u_{h, j}, I_{2 h} u_{h, j}\right)}{\left(I_{2 h} u_{h, j}, I_{2 h} u_{h, j}\right)} .
$$

Table 3 and Table 4 confirm the estimation obtained in Theorem 4 for eigenvalues. 
Table 1: The error $\left\|u_{j}-u_{h, j}\right\|_{2, \Omega}$

Table 2: The error $\left\|u_{j}-I_{2 h} u_{h, j}\right\|_{2, \Omega}$

\begin{tabular}{c|c|c|c}
$N_{e}$ & $j=1$ & $j=2$ & $j=3$ \\
\hline 8 & 0.88 & 0.16 & $1.1 \times 10^{-2}$ \\
16 & 0.19 & $1.9 \times 10^{-2}$ & $1.3 \times 10^{-3}$ \\
32 & $2 \times 10^{-2}$ & $2.5 \times 10^{-3}$ & $1.7 \times 10^{-4}$
\end{tabular}

Table 3: The error $\left|\lambda_{j}-\lambda_{h, j}\right|$

\begin{tabular}{c|c|c|c}
$N_{e}$ & $j=1$ & $j=2$ & $j=3$ \\
\hline 8 & $6.8 \times 10^{-2}$ & $1.9 \times 10^{-3}$ & $3.6 \times 10^{-5}$ \\
16 & $2.6 \times 10^{-2}$ & $7.1 \times 10^{-3}$ & $2.3 \times 10^{-5}$ \\
32 & $9.5 \times 10^{-3}$ & $2.9 \times 10^{-4}$ & $9.1 \times 10^{-6}$
\end{tabular}

Table 4: The error $\left|\lambda_{j}-\widetilde{\lambda}_{h, j}\right|$

\begin{tabular}{c|c|c|cc|c|c|c}
$N_{e}$ & $j=1$ & $j=2$ & $j=3$ & $N_{e}$ & $j=1$ & $j=2$ & $j=3$ \\
\hline 8 & 0.78 & $2.5 \times 10^{-2}$ & $1.2 \times 10^{-4}$ & 4 & 2.7 & $1.8 \times 10^{-2}$ & $2.4 \times 10^{-6}$ \\
16 & $3.8 \times 10^{-2}$ & $3.9 \times 10^{-4}$ & $1.8 \times 10^{-6}$ & 8 & $4.2 \times 10^{-3}$ & $2.4 \times 10^{-6}$ & $3.1 \times 10^{-9}$ \\
32 & $4.1 \times 10^{-4}$ & $6.4 \times 10^{-6}$ & $2.9 \times 10^{-8}$ & 16 & $7 \times 10^{-4}$ & $4.1 \times 10^{-9}$ & $4.4 \times 10^{-10}$
\end{tabular}

\section{CONCLUDING REMARKS}

The ultraconvergence results for the approximation of some planar fourth-order elliptic problems enable us to conclude that:

- We present an ultraconvergent patch-recovery method applied to biharmonic problems and using rectangular finite elements. This method requires some sort of elaboration but in return gives an effective and simple algorithm;

- The interpolated finite elements described in the paper are in higher preference as compared to the standard rectangular elements. They gain two order higher convergence and even four order when they are applied to eigenvalue problem;

- In order to concentrate on the local recovery procedures, it is assumed that the exact solutions of the problems considered here are sufficiently smooth. In other words, the discussion of the singularity and boundary behaviour is omitted;

- Application of the patch-recovery method to eigenvalue problems is related to the supercloseness between the elliptic (Ritz) projection and the approximate eigenfunction (see Lemma 3);

- This paper employs a kind of analysis that can be applied to more general fourthorder elliptic operator. It is also open to generalizations to higher dimension tensor product spaces, especially for brick elements in three dimensions. These will be addressed in a separate paper. 


\section{REFERENCES}

[1] Ainswort M. and Oden J.T., A Posteriori Error Estimation in Finite Element Analysis. Wiley Interscience, New York, 2000.

[2] A.B. Andreev, Supercloseness between the elliptic projection and the approximate eigenfunction and its application to a postprocessing of finite element eigenvalue problems, LNCS 3401, Springer-Verlag, 100-107, 2005.

[3] A. B. Andreev, T. T. Dimov and M. R. Racheva, One-dimensional patch-recovery finite element method for fourth-order elliptic problems, LNCS 3401, SpringerVerlag, 110 117, 2004.

[4] I. Babuška, J. Osborn, Eigenvalue Problems, Handbook of Numer. Anal., Vol.II, North-Holland, Amsterdam, 1991.

[5] P.G. Ciarlet, The Finite Element Method for Elliptic Problems, North-Holland, Amsterdam, New York, Oxford, 1978.

[6] Li B., Zhang Z., Analysis of a class of superconvergence patch-recovery techniques for linear and bilinear finite elements, Numer. Methods for Partial Differential Equations 15 (1999), 151-167.

[7] Liu Q., Yan N. and Zhou A., A rectangular test for interpolated finite elements, Proceedings of Systems Science \& Systems Engineering, Culture Publish Co., 1991, 217-229.

[8] G. Strang, G.J. Fix, An Analysis of the Finite Elemente Method, Prentice-Hall, Englewood Cliffs, NJ, 1973.

[9] Thomee V., Galerkin Finite Element Methods for Parabolic Problems, Springer, 1997.

[10] T.D. Todorov and M.R. Racheva, Applications of a two-level method for isoparametric iterative scheme for solving elliptic problems, Notes on Numerical Fluid Mechanics, Springer-Verlag, Vol. 73, 196-203, 2000.

[11] Verfürth T., A Posteriori Error Estimation and Adaptive Mesh Refinement Techniques, Teubner Skripten zur Numerik, B. G. Teubner, Stuttgart, 1995.

[12] L.B. Wahlbin, Superconvergence in Galerkin FEM, Springer-Verlag, Berlin, 1995.

[13] Wang J., A superconvergence analysis for finite element solutions by the leastsquares surface fitting on irregular meshes for smooth problems, J. Math. Study 33(3) (2000).

[14] Zhimin Zhang, Runchang Lin, Ultraconvergence of ZZ patch recovery at mesh symmetry points, Numer. Math. (2003) 95, 781-801.

[15] Zhu Q. and Zhao Q., SPR technique and finite element correction, Numer. Math. (2003) 96, 185-196. 
[16] O.C. Zienkiewicz and J.Z. Zhu, The superconvergence patch-recovery and a posteriori error estimates. Part 1: The recovery technique, Int. J. Numer. Methods, Eng., 33, 1331-1364 (1992).

[17] O.C. Zienkiewicz and J.Z. Zhu, The superconvergence patch-recovery and a posteriori error estimates. Part 2: Error estimates and adaptivity, Int. J. Numer. Methods, Eng., 33, 1365-1382 (1992). 